\title{
A Quantitative and Qualitative Analysis of Strategy of Translation Based on TAPs
}

\author{
Hulin Zhao \\ Faculty of International Studies, Henan Normal University, Xinxiang City, China \\ Email address: \\ 819235660@qq.com

\section{To cite this article:} \\ Hulin Zhao. A Quantitative and Qualitative Analysis of Strategy of Translation Based on TAPs. International Journal of Language and \\ Linguistics. Vol. 7, No. 5, 2019, pp. 182-187. doi: 10.11648/j.ij11.20190705.11
}

Received: April 11, 2019; Accepted: July 16, 2019; Published: August 5, 2019

\begin{abstract}
The strategy of translation is understood as equivalent to the problem-solving strategy in this research. The article adopts a mixed approach to the contrastive analysis of Chinese-English (C-E) and English-Chinese (E-C) translation strategy based on think-aloud protocols. Research question is: What similarities and differences can be found between C-E and E-C translation strategy? It is hoped that research findings can shed some light on the translation strategy research and education of student translators' translation competence.
\end{abstract}

Keywords: Translation Strategy, Student Translator, Translation Competence

\section{Previous Research on Translation Strategy}

The German scholar Lörscher has carried out a series of studies on translation strategies via Think-aloud method (TAM). Lörscher's systematic think-aloud approach to the translation process identifies 20 strategies. Moreover, his research findings demonstrate that professional translators differ from language learners in translation strategy. To be specific, the former pay more attention to whether the style and text typology of the translated versions are adequate in the target language; while the latter focus on the translation of words or sentences, neglecting the general textual features of the translated versions. Other similar studies verify Lörscher's findings [1-3]. Furthermore, the scholars also manage to differentiate among various translation strategies [4].

Similarly, Kiraly [5] makes such a lengthy classification of the translation strategies as accept interim solution, attempt syntactic reconstruction, back translate, break off attempt, break off translation and start over, employ mnemonic aid, identify problem, dictionary search, make extra-linguistic judgment, make intuitive acceptability judgment, monitor for accuracy, dictionary solution, proposed dictionary solution, recontextualize, reduce meaning, refer to translation expectation, rephrase SL segment, uncertainty regarding acceptability, uncontrolled interim unit and unsuccessful dictionary search. The above is 20 strategies identified by Kiraly through his think-aloud approach to the German-English translation process. Arlene \& Rossana (2019) analyses the impact of two different machine translation outputs on the cognitive effort required to post-edit machine-translated metaphors by means of eye tracking and think-aloud protocols [6].

Researchers in China with TAM probe into the use of cognitive and meta-cognitive strategies while three subjects translate into Chinese English sentences of cause-effect relations and sentences of double negation. With 20 juniors of English majors in China as the research subject, Wen and Yin study the use of translation strategy via TAM [7]. The research compares its divergence of students with low and high grades. In addition, the differences of Chinese and German students and in four translation phases of analysis, transfer, restructure and test are conducted, too.

\section{Reliability and Validity of TAM}

\subsection{Debates on Its Reliability and Validity}

TAM refers to a kind of data-collecting method that asks the participants to verbalize simultaneously what they are thinking about as they are working on a particular task. Borrowed from psychology and increasingly applied into translation studies, it is one method that is believed to 
demystify the thought process of the human brain. It has been one of the empirical methods used in the investigation of the psychological aspect of the translation process. Participants who face the task of producing a translation are asked to speak aloud whatever comes into their minds while they are working on it. Their verbalizations, also called think-aloud protocols (TAPs), are recorded and then meticulously analyzed. Consequently, inferences are actually indirectly made about the cognitive processes that produce the verbalizations.

TAM is a well defined methodology, underlying which is the assumption that "human cognition is information processing" [8]. And translation is also assumed to be one of the general phenomena of the information processing, which takes place in the working memory of the human brain and in turn, what is stored in it can be verbalized [9]. A further assumption is that the think-aloud itself does not "interfere with the cognitive process itself, the only effect being to slow down the task performance" [10]. In this sense, it is believed what the participants verbalize reflects or even stands for an accurate record of what they think in reality.

On the one hand, a number of studies have applied TAM into translation research; on the other, a multitude of other studies, however, have pointed out the problems in the application of TAM [11]. The problems identified can be summarized as 1) the think-aloud data gathered through TAM may be incomplete; 2) it is not plain what influences the methodology of TAM itself will exert on the translation process; 3) how the two translation modes of verbal report and translation interfere with each other; 4) how the validity and reliability of the experiment is assured.

In response to the controversies, many other researches contend that TAM can be relied on to disclose the translation process. Benefiting from the process model proposed by Ericsson and Simon, Bernardini (ibid) holds that "only concurrent verbalization of thoughts [TAPs] can be claimed to exhaustively reflect the mental state of a participant carrying out a relatively long task (let us say, longer than ten seconds)"; a single think-aloud will not distort the mental state.

\subsection{Safeguards of Its Reliability and Validity}

TAM is said to be one of the most powerful means of revealing the translation process. It can yield rich data, provided that the procedure of data-collection, data coding and analysis observes certain principles. However, no method comes without its deficiencies.

Arguably the biggest drawback in using TAM is that the entire process of collecting and analyzing think-aloud data consumes a great deal of time and energy. Moreover, the verbal report may, to some extent, slow down the translation, so the participants often take more time than usual to complete the translation task. Last but not least, it is still not clear till today how the verbalization and translation work with each other. Anyhow, the methodology will not lose its advantage even if it still needs improving. And the researcher has made painstaking effort to minimize the impact of the external factors and in this way, to maximize the validity and reliability of the think-aloud procedure, thus the quality of the collected data can be guaranteed.

Building on Guba's criteria for ensuring the quality of naturalistic [qualitative] inquiries, Li Defeng outlines his 8 safeguards for the trustworthiness of TAM [12]. Jointly combining the safeguards discussed by Li and the nature of the current research, the author summarized the safeguards as shown in Table 1. The current research obeys the table to conduct the research design.

Table 1. Safeguards for the trustworthiness of the research design.

\begin{tabular}{ll}
\hline Stage of the research & Safeguards \\
\hline & Voluntary participation \\
& Anonymity assurance \\
& Purposeful sampling \\
Data collection & Triangulation \\
& Prolonged engagement \\
& (Near-) Natural situation \\
& Peer debriefing, stepwise replication \\
Data analysis & Member checks \\
Reporting & Thick description \\
Application & Refrain from generalizing \\
\hline
\end{tabular}

\section{Research Design}

\subsection{Participants Selection}

11 English major juniors are selected according to their TEM (Test for English Majors) 4 score, scores of C-E and E-C translation, and their overall rank of the $5^{\text {th }}$ semester. They volunteer to participate in the experiment. 7 of them survive the training session and are considered to be eligible for the think-aloud experiment, three of which are males, the rest females. The participants are assured of their anonymity. All the participants are top students in the same grade, for the simple fact that to think-aloud poses great challenges to the participants and it is out of the reach of students with poor performance. Besides, the thesis will not make any comparison between top students and underdeveloped students.

\subsection{Participants Training}

The participants should be well-informed about what they are expected to do, the methodology itself as well as the reasons for conducting; therefore, the following instructions are delivered. The training material used at this stage is similar to that at the think-aloud phase.

In this research, I take interest in what you think about as you carry out the tasks I am going to give you. To do this, you are asked to think aloud as you work through the tasks. By "think aloud" I mean that I want you to say everything that you are thinking from the very first beginning you start the task until you finish it. Please do not explain your thought process or why you have such thought. You are advised to speak constantly during the whole process. The current experiment is an essential part of my MA thesis which hopes to provide some suggestions on the TEM 8 translation test design, test preparation and the translation teaching and learning. 


\subsection{Test Material}

The test material employed in this research is picked up from the sample paper of TEM 8 [13], which is considered "ideal sample" [14]. It covers C-E and E-C translation. It is chosen obeying by the following principles:

1) The test material should mirror the test itself.

2) It should be neither too difficult nor too easy for the participants to complete.

3) The test types should include selections both from literature works and English periodicals.

\subsection{Pilot Study}

The think-aloud experiment design goes through trials on two students with the research interest in translation prior to its formal exercise. The researcher finds that major problems to tackle in carrying out the experiment are 1) the instruction should be well worded in the hope of not misleading the participants; 2) the experiment goes against the normal state of the translation behavior, therefore, the participants need time to get accustomed to it; 3 ) outgoing and open-minded students tend to verbalize more than those being introverted.

\subsection{TAPs Collection}

The sheet of instructions is shown to the participants once more. And the researcher briefs them on the think-aloud procedure, and prompts in case that their pause intervals exceed 5 seconds. Then the participants are provided with some practice tasks such as the multiplicity by numbers and the translation task. Finally comes the actual think-aloud phase.

During this phase, the participants are given the translation materials chosen from a TEM 8 sample test paper. They are asked to write down their translated versions, and at the same time, express what is coming into their brains. And the voice-recording device is available to keep record of what they have verbalized. Thus, two types of data will be obtained as soon as the experiment is completed; one is the translated version, the other think-aloud protocols. Each participant performs his or her task individually in a small secluded classroom. There is no time limit and the use of dictionaries is not allowed even if the participants encounter new words or phrases.

\subsection{TAPs Transcription}

The initial data transcription, including both the think-aloud data and the translated version, is assisted by some classmates of the researcher, but it is the researcher himself who edits and determines the final version. Not only the participants' words but also sighs, laughs, etc. are transcribed to obtain the full and comprehensive data.

\subsection{TAPs Coding}

Data coding is crucial to abstracting useful and valid information from think-aloud protocols. A good coding scheme achieves a balance between specificity and generalizability. That is to say, the coding scheme can be neither too general nor too detailed, which otherwise would either fail to capture adequately the cognitive activity involved in carrying out a task or not succeed in representing the typical behavior.

The coding scheme shapes and constrains the inferences that may be drawn from the data. Data coding involves developing a coding scheme, determining the unit for analysis and segmenting the protocols. A unit for analysis will usually comprise a phrase, clause or sentence. As for the segmentation of the protocols, each segment should be representative of a single specific process. The researcher intends to encode the data based on 3 types of data, self-report, self-evaluation and self-revelation, proposed by Cohen [15]. The coding scheme is illustrated at length in the following part. One segment which is coded between the sign // represents a single process.

Based on the previous researches on the translation strategy, the thesis makes out the following 22 strategy indicators as the criteria to classify the strategies of the current research. It is shown in the table below.

Table 2. Coding scheme of ST.

\begin{tabular}{|c|c|c|}
\hline Codes & Meanings & Explanations \\
\hline ST1 & Interim solution & give tentative TL (target language) versions \\
\hline ST2 & Accept interim solution & make choice from the tentative versions \\
\hline ST3 & Syntactic reconstruction & modify the syntactic structure of the translation \\
\hline ST4 & Back translate & translate the TL version back to a SL version \\
\hline ST5 & Break off attempt & stop temporarily the attempt \\
\hline ST6 & Break off and start over & continue to try and think from the broken part \\
\hline ST7 & Employ mnemonic aid & search for methods and solutions in memory \\
\hline ST9 & Make intralinguistic judgment & use knowledge of the SL (source language) text \\
\hline ST10 & Make extralinguistic judgment & use general world knowledge and context \\
\hline ST11 & Make acceptability judgment & judge the intuitive solution \\
\hline ST12 & Monitor TL accuracy & Judge the rightness of the TL translation \\
\hline ST13 & Reduce meaning & give up some selective solutions \\
\hline ST14 & Refer to translation expectation & translation target \\
\hline ST15 & Rephrase SL segment & paraphrase SL segment \\
\hline ST17 & Uncontrolled production & automatic solution to SL segment \\
\hline ST18 & Read SL segment & make reading of the SL text \\
\hline ST19 & Self-correction & correct what one has just said \\
\hline
\end{tabular}




\begin{tabular}{lll}
\hline Codes & Meanings & Explanations \\
\hline ST20 & Read the whole SL & make reading of the entire text \\
ST21 & Make grammatical judgment & analyze the SL segment in grammar \\
ST22 & Solution to the problem & find answer to question \\
\hline
\end{tabular}

\section{Research Results}

The data analysis has two parts included, qualitative and quantitative analysis. The qualitative analysis primarily relies on the case study, selecting two samples from the 7 sets of protocols. Moreover, the analysis adopts a bottom-up method to search for whether any trend can be traced. The existing research is primarily qualitative, and in this sense, its theme should be the case analysis of the protocols. Besides, the case analysis is supplemented by the quantitative analysis of the whole set of data produced by all the 7 participants.

\subsection{Quantitative Analysis of ST}

Table 3. ST analysis.

\begin{tabular}{lllllllllllllllllllllllll}
\hline ST Type & & $\mathbf{1}$ & $\mathbf{2}$ & $\mathbf{3}$ & $\mathbf{4}$ & $\mathbf{5}$ & $\mathbf{6}$ & $\mathbf{7}$ & $\mathbf{8}$ & $\mathbf{9}$ & $\mathbf{1 0}$ & $\mathbf{1 1}$ & $\mathbf{1 2}$ & $\mathbf{1 3}$ & $\mathbf{1 4}$ & $\mathbf{1 5}$ & $\mathbf{1 6}$ & $\mathbf{1 7}$ & $\mathbf{1 8}$ & $\mathbf{1 9}$ & $\mathbf{2 0}$ & $\mathbf{2 1}$ & $\mathbf{2 2}$ \\
\hline C-E & $\mathrm{N}$ & 34 & 34 & 21 & 4 & 14 & 14 & 11 & 36 & 4 & 0 & 125 & 11 & 17 & 1 & 54 & 13 & 11 & 56 & 42 & 2 & 41 & 36 \\
translation & $\%$ & 6 & 6 & 3.6 & 0.6 & 2.4 & 2.4 & 2 & 6 & 0.6 & 0 & 21.5 & 2 & 3 & 0.1 & 9 & 2.2 & 2 & 9.6 & 7 & 0.3 & 7 & 6 \\
E-C & $\mathrm{N}$ & 39 & 39 & 26 & 0 & 14 & 14 & 6 & 29 & 9 & 0 & 123 & 44 & 16 & 0 & 5 & 36 & 82 & 46 & 75 & 5 & 16 & 29 \\
translation & $\%$ & 6 & 6 & 4 & 0 & 2 & 2 & 1 & 4.4 & 1.4 & 0 & 19 & 6.7 & 2.4 & 0 & 0.7 & 5.5 & 12.5 & 7 & 11 & 0.7 & 2.4 & 4.4 \\
\hline
\end{tabular}

In total, the strategy indicators in the C-E translation (581) are a little bit less than those in the E-C translation (658). Almost all the STs have ever been used except ST10 which is not resorted to at all in the C-E translation or the E-C translation. ST4 and ST14 are not used in the latter, either.

1) There is no obvious difference in the use of ST1-ST2, ST3, ST5-ST6, ST7, ST9 and ST13.

2) The first five strategies of translation in the former are ST11, ST18, ST19, ST15 and ST21, while the last five are ST10, ST14, ST20, ST4 and ST9. By contrast, strategies like ST11, ST17, ST19, ST18 and ST12 rank the first five in the E-C translation; whereas ST10, ST14, ST4, ST15 and ST20 rank the last five.

3) ST4, ST10 and ST14 have never been applied in the E-C translation, while ST10 alone is not visible in the C-E translation.

4) In both types of translation, ST11 forms the most frequently adopted strategy, accounting for $21.5 \%$ in the C-E translation and $19 \%$ in the E-C translation. It can be interpreted that the translation process aims at the actual operation of the source text, or the translation process is a problem-solving process.

5) The number of ST12 in the C-E translation is 11, a percentage of $2 \%$, while it is 44 in the E-C translation, a percentage of $6.7 \%$. It demonstrates that the participant has an easy time in monitoring the production of the translated version when the target language is his or her mother tongue. It is the same with ST19 and ST17 that the participant is more likely to make corrections and to automatically produce the translation. In the C-E translation, the frequency of ST19 and ST17 stands at 42 and $7 \%, 11$ and $\%$; nevertheless, in the E-C translation that of ST19 and ST17 is 75 and $11 \%, 82$ and $12.5 \%$. It is another case for ST16, even though the mother tongue acts as the target language. The measure of 36 and $5.5 \%$ in the E-C translation outweighs that of 13 and $2.2 \%$ in the $\mathrm{C}-\mathrm{E}$ translation. When the mother tongue works as the source language, the participant tends to paraphrase the source text. The point can be testified by the use of ST15, 54 in number and $9 \%$ in percent in the C-E translation and 5 in number and no more than $1 \%$ in percent in the opposite translation.

6) In terms of ST18, the proportion in the C-E translation process is more than $2 \%$ higher than that in the E-C translation. The result is not in accordance with Wen and Yin's (2010) research finding that when the source language is the native language, the participant does not frequently use the strategy of reading ST segment. It is guessed that the difficulty of the test material, the participant's translation skills or the direction of the translation lie behind the appearance of the phenomenon in the current research. In other words, the difficulty level of the C-E translation is higher than that of the E-C translation; the participant has a better command over the E-C translation than the C-E translation; or the participant has less trouble in translation into the native language than from it. The higher difficulty level of the $\mathrm{C}-\mathrm{E}$ translation can be mirrored by ST8-ST22, whose frequency is higher than that of the E-C translation.

\subsection{Qualitative Analysis of ST}

All Chinese segments in this part have been expressed in English, but I don't mark them yellow.

This part makes a detailed categorization of each segment produced by participant 2 (P2). It is carried out with the original verbalization, the analysis and the categorization of ST. The analysis of the protocols illustrates that the translation strategies used by P2 have the following features.

The participant browses through the material before her translation and the protocols end with the revision of the translation. Segment 1 to segment 4 mark the pre-read part, which initiates and concludes with "the sentence is used to describe the features of my supervisor", and "it generally means my supervisor is very strict with his students, but 
appreciates the diligence of Asian students", respectively. Segment 5 to segment 100 comprise the theme part of the segments, which begins and ends with reading of the material "Asian American" and modification of the translation "gain, have are two commonly used words, so I will choose the word 'obtain', meaning making efforts to strive for the doctoral degree", separately. Segment 101 to 119 make up the revision part, which begins and ends with reading of the SL segment "My supervisor is an Asian American" and her self-judgment of the translation "It is acceptable to revise like this".

Some translation strategies tend to appear in pairs. Strategies like ST1-ST2, ST5-ST6, ST8-ST22 more often than not appear in groups. For instance, segment 7 and 8 form a pair, which first lists several choices of the translation of Asian American and then one choice is made; segment 13 and 16 dealing with the translation of bad tempered comprise the other pair ST5-ST6. The participant makes attempts to translate the phrase but finds no solutions and resumes the translation at segment 16 . Segment 52 and 53 are a case in point to witness the simultaneous appearance of ST8-ST22. In addition, the presence of ST5-ST6 also proves that the translation process is non-linear but recursive, for the simple fact that the participant goes back to the earlier translation.

When no obstacles turn up in dealing with translation, the participant often resorts to the ST18/ST15-ST11-ST1-ST2 pattern in strategy use. It means that the participant first reads or paraphrases the SL segment, then searches for the translated version of the SL segment or gives several selections before the ultimate choice is made. Some actual translation process does not strictly observe the pattern. For instance, segment 5 starts with the reading of the SL segment, but omits the ST11 phase and goes directly to ST1, and concludes with ST2. Besides, segment 5 to 7 are interwoven with another strategy ST7. However, when obstacles are present, the ST18/ST15-ST11-ST5-ST6 pattern is applicable. This point can be confirmed by segment 34 to 39 , which initiate with the reading of the SL segment, stop temporarily the translation and proceed from where pauses. The process is intermitted with ST3 and ST15.

In terms of $\mathrm{ST}$, the protocols in the E-C translation process have the following characteristics:

The participant pre-reads the test material, then carries out the translation and at last revises the translation. Segment 1 to 22 mark the pre-reading phase, segment 23 to 120 for the actual translation phase, segment 121 to 152 for the revision phase.

Strategies like ST1-ST2, ST5-ST6, ST8-ST22 more often than not appear in pair, such as segment 26 and 29, segment 40 and 41, and segment 69 and 72.

The ST17/ST11-ST19 pattern in general is prevalent in dealing with the E-C translation. It means that the participant either automatically produces the translation or carefully searches for the translation, which is usually followed by the modification of the translation. For instance, segment 77 is an automatic production of the translation, segment 78 the search for the translation. Similarly, segment 79 and 80 repeat the above cycle. And the whole process is ended with segment 81 of the modification of the translation. When obstacles are found in the process, however, the participant breaks off the translation and finally resumes it, thus the ST17/ST11-ST19-ST5-ST6 pattern is adopted. The point can de shown by segment 36 to 41 . Otherwise, the ST17/ST11-ST19-ST1-ST2 pattern is applicable to some segments in the protocols. Segment 23 to 29 are a case in point.

In comparison, the qualitative analysis of the protocols in the two kinds of translation process illustrates that they share the following similarities in ST.

1) The translation process looks like one with the pre-reading phase, the translating phase and the revising phase.

2) Some pair patterns appear like ST1-ST2, ST5-ST6 and ST8-ST22.

Besides, varied ST patterns are found in the protocols. The ST18/ST15-ST11 pattern is common in the C-E translation process, while the ST17/ST11-ST19 pattern occupies a dominant role in the $\mathrm{E}-\mathrm{C}$ translation process.

\section{Discussion}

The rich strategy segments produced by participants show, to some extent, their strategy awareness. That is to say, they consciously or unconsciously realize that they can resort to strategies to solve the problems that they encounter during the translation process.

Compared with the frequent use of ST12 and ST17 in the E-C translation, the strategy of ST15 and ST21 is used more frequently in the C-E translation. In the E-C translation, participants goes deeper into the translated text, while in the C-E translation, participants still stops at the understanding of the material. It can be said that the strategy use in the former translation enjoys a higher level than in the latter. It may illustrate from another aspect that the C-E translation is more difficult than the E-C translation. The above brief discussion is followed by some suggestions and implications.

The far less frequently used ST10, ST20 and ST9 reveals that both the textual and extratextual knowledge are not fully exercised. Besides, the almost absence of ST14 also shows that participants have no awareness to grapple with the translation from the macro perspective. Only when problems appear during the translation, will the participant depend on the context to guess how to deal with some word, phrase or sentence. The translator should have the concept of discourse and make discourse smooth his or her translation.

\section{Conclusion}

The qualitative analysis shows that the translation process in general is non-linear but recursive. It is not the novel finding of the current research but the further testification.

In terms of ST, 1) there is no obvious difference in the use of ST1-ST2, ST3, ST5-ST6, ST7, ST9 and ST13. 2) different 
strategy patterns appear in the translation process, ST18/ST15-ST11 pattern in the C-E translation process, and the ST17/ST11-ST19 pattern in the E-C translation process. 3) The first five strategies of translation in the former are ST11, ST18, ST19, ST15 and ST21, while the last five are ST10, ST14, ST20, ST4 and ST9. By contrast, strategies like ST11, ST17, ST19, ST18 and ST12 rank the first five in the E-C translation; whereas ST10, ST14, ST4, ST15 and ST20 rank the last five. 4) ST4, ST10 and ST14 have never been applied in the E-C translation, while ST10 alone is not visible in the C-E translation. 5) In both types of translation, ST11 forms the most frequently adopted strategy. 6) ST12, ST17 and ST19 are used more frequently in the E-C translation than in the C-E translation. It demonstrates that it is easier for the participants to monitor the production of the translated version, make corrections and automatically produce the translation in the E-C translation when the target language is his or her mother tongue. It is another case for ST16, even though the mother tongue acts as the target language. It is adopted more often in the $\mathrm{C}$-E translation than otherwise. In the C-E translation when the mother tongue works as the source language, the participant tends to paraphrase the source text. The point can be testified by the use of ST15, 54 in number and $9 \%$ in percent in the $\mathrm{C}$-E translation and 5 in number and no more than $1 \%$ in percent in the opposite translation. 7) As for ST18, the proportion in the $\mathrm{C}-\mathrm{E}$ translation process is more than $2 \%$ higher than that in the E-C translation.

The future studies are advised to be undergone from a larger perspective such as larger samples, more translation aspects. Moreover, efforts should be made to improve TAM itself.

\section{Acknowledgements}

The research is sponsored by Educational Research Project of China National Committee for Translation \& Interpreting Education (No. MTIJZW201807), Project for Young Scholars of Henan Normal University and Startup Project for Recruit with Ph. D Degree of Henan Normal University (qd17156).

\section{References}

[1] Fraser, Janet. Public Accounts: Using Verbal Protocols to Investigate Community Translators [J]. Applied Linguistics, 1993 (4): 352-353.
[2] Fraser, Janet. Translating Practice into Theory: A Practical Study of Quality in Translator Training [A]. Catriona Picken (ed.). ITI Conference 7 Proceedings [C]. London: Institute of Translation and Interpreting. 1994.

[3] Olalla-Soler, Christian. Using Translation Strategies to Solve Cultural Translation Problems [J]. Perspectives, 2019 (3): 367-388.

[4] Séguínot, Candace. Some Thoughts about Think-aloud Protocols [J]. Target, 1996 (1): 75-95.

[5] Kiraly, D. Think-aloud protocols and construction of a professional translator self-concept [A]. Joseph Danks, et al. (eds.). Cognitive Processes in Translation and Interpreting (pp. 137-160) [C]. Thousand Oaks, California: Sage Publications. 1997.

[6] Arlene, K. \& C, Rossana. Investigating the Post-editing Effort Associated with Machine-translated Metaphors [J]. Journal of Specialised Translation, 2019 (31): 38-59.

[7] Wen, Jun. \& Yin, Ling. Translation Strategies of Chinese English Majors [J]. Journal of PLA University of Foreign Languages, 2010 (4): 75-80.

[8] Ericsson A. \& Simon, A. Protocol Analysis (2nd ed.) [M]. Cambridge, MA: MIT Press. 1993.

[9] Ericsson A. \& Simon, A. Verbal Protocols as Data [M]. Cambridge, MA: MIT Press. 1984.

[10] Bernardini, Silvia. Think-aloud Protocols in Translation Research [J]. Target, 2001 (2): 241-263.

[11] Jääskeläinen, Ritta. Think-aloud Protocol Studies into Translation: An annotated bibliography [J]. Target, 2002 (1): 107-136.

[12] Li, Defeng. Trustworthiness of Think-aloud Protocols in the Study of Translation Processes [J]. International Journal of Applied Linguistics, 2004 (3): 301-313.

[13] Zou, Shen. Sample Test Papers for TEM 8 [M]. Shanghai: Shanghai Foreign Languages Education Press. 2005.

[14] Zheng, Binghan. The Triangulation Module in Process-oriented Translation Studies [J]. Shanghai Journal of Translators, 2008 (3): 36-41.

[15] Cohen, Andrew. Studying Second-Language Learning Strategies: How Do We Get the Information? [J]. Applied Linguistics, 1984 (5): 101-112. 